\title{
An experimental study of strategy development
}

\author{
ELIZABETH J. NEWTON and MAXWELL J. ROBERTS \\ University of Essex, Colchester, England
}

\begin{abstract}
Three experiments are reported in which the strategy usage for a compass point directions task was investigated. For this task, people may use either an efficient cancellation strategy or an inefficient spatial strategy (see Roberts, Gilmore, \& Wood, 1997). Experiment 1, together with Experiment 2, showed that users of cancellation are unlikely to have explicit prior knowledge of this strategy; instead they discover it as a result of experience with the task. Experiment 1 also showed that strategy selection is unlikely to be based on stylistic preferences, and that reducing the cognitive demands of the task does not increase the discovery of cancellation. Experiment 3 showed that evaluation of strategy validity is also of importance: Unsuccessful evaluation may prevent the selection of a newly discovered strategy, even if it is the more efficient method.
\end{abstract}

The study of individual differences in strategy usage is gaining importance, although the majority of research has focused on children. Here many researchers (e.g., Siegler, 1996) argue that performance can be characterized as the continual development and refinement of numerous task-specific strategies. If it is accepted that differences in strategy usage are an important aspect of adult cognition too, then any research that fails to account for these will be incomplete. Until recently, there has been little attempt to integrate findings in this topic (for exceptions, see Crowley, Shrager, \& Siegler, 1997; Kuhn \& Pearsall, 1998). One consequence of this is that there is disagreement even with the definitions of basic terms such as strategy. For the studies to be described in the present article, Siegler and Jenkins's (1989) definition will be preferred: A strategy is "any procedure that is non-obligatory and goal directed" (p. 11). Hence, a strategy is any set of self-contained cognitive processes that can be dispensed with in favor of alternatives. This definition precludes any assumptions concerning (1) whether or not processes are hard wired, and (2) the phenomenological status of the processes associated with change.

Strategy development - the change in strategy usage with time - can be broken down into at least two components. On the one hand, there is the need to explain strategy selection. Accounts differ in the extent to which people are said to be sensitive to current task demands and performance. At one extreme, cognitive style accounts posit that choice of strategy is determined by a tendency, or preference, to represent and process information in a

We thank Tim German and two anonymous referees for their comments on earlier versions of the manuscript, and Steve Avons for his comments and suggestions when planning the research, as well as his assistance when collecting the data. Correspondence should be addressed to M. J. Roberts, Department of Psychology, University of Essex, Wivenhoe Park, Colchester CO4 3SQ, England (e-mail: mjr@ essex. ac.uk). particular way (e.g., Sternberg, 1997). One example is the visualizer-verbalizer style (e.g., Riding \& Rayner, 1998). However, selected strategies will not be adaptive where the stylistic preference is inappropriate for a particular task. Other accounts posit that task characteristics and/or current and past performance have far more substantial roles to play. For example, Shrager and Siegler's (1998) SCADS model incorporates a mechanism for selecting between strategies. This is based upon their general success, and their relative success at specific problems or types of problem. This model explains why newly discovered superior strategies are often generalized slowly. With little experience, there can be little associated success.

Although strategy selection is an important component of strategy development, any account is incomplete without a mechanism to explain strategy possession and acquisition. An extreme knowledge-based account of this would suggest that for any task, the strategy repertoire of an individual is determined entirely by past experience. This stance is derived primarily from studies into expertise (e.g., Ericsson \& Lehmann, 1996). These show that experts perform better than novices, not because of superior general abilities, but because of extensive domainspecific knowledge. More controversial is to infer additionally from this that for any task, those who choose the best strategies must, without exception, have gained them from prior experience by having encountered the task, or related tasks, previously. In other words, "the study of individual differences is closely tied to the study of learning and transfer of training" (Simon, 1990, p. 15; see also Schiano, Cooper, Glaser, \& Zhang, 1989). This account offers a rather static view, and many researchers instead focus on how new strategies arise as a result of current experience gained while one is interacting with a task (e.g., Crowley et al., 1997; Roberts, Gilmore, \& Wood, 1997). Here, there is the need to take account of what can be learned about a task from the way in which information is represented and manipulated, and of how this is affected by individual differences. We need to understand 
how new strategies are discovered, and which events encourage this.

Broadly, accounts of how problem solving events lead to strategy discovery are either failure based or success based. For example, VanLehn (1988) has suggested that discovery is triggered as a result of the failure of a current strategy to generate a satisfactory answer when one is solving a problem - that is, an impasse. However, Siegler and Jenkins (1989) found that impasse problems for addition could only change the current problem-solving strategy to one already known. They did not trigger the generation of new strategies for overcoming them. Successdriven strategy discovery has been implicated by numerous past studies (e.g., Crowley et al., 1997; Karmiloff-Smith, 1992; Roberts et al., 1997). For example, people who discover short-cut strategies for solving linear syllogisms are initially better at solving them by using imagery strategies (e.g., Wood, 1978), and Galotti, Baron, and Sabini (1986) found that good reasoners at categorical syllogisms were the more likely to discover short-cuts. The key here appears to be that a successful problem solver is able to represent information accurately and stably; and from this, such a person is better able to detect regularities, delete redundant steps, and hence discover less cumbersome, more elegant strategies.

\section{The Directions Task}

Roberts et al. (1997) reported a series of studies in which compass point directions tasks were investigated. Here, people are given a series of trials, each consisting of sets of compass point directions (e.g. one step north, one step east, one step south, one step south, one step east). The task is to determine where a person would end up, relative to the starting point, after taking them. When all steps are presented at once on a single screen (parallel presentation), the majority of people report the use of one of two strategies. For the spatial strategy, a full representation of the path is generated, and the final bearing is read from this. For the cancellation strategy, opposite directions are cancelled, with those that remain constituting the correct answer. The spatial strategy is generally slow, inaccurate, and occasionally stressful to execute, but it still tends to be used by many people. Cancellation is generally faster and more accurate. When spatial strategy users are instructed to use cancellation, their rapid improvement suggests that the latter is not merely a highly practiced spatial strategy. The directions task has several properties that make it useful for investigating strategy development. The task is novel, yet its requirements are easy to understand. There is a close relationship between reported strategy and performance, indicating that the strategies are highly available to conscious recall, even retrospectively, thus reducing the need for an intensive and extensive microgenetic study (e.g., Siegler \& Jenkins, 1989). Unlike in the tasks studied elsewhere (e.g., those studied by Kuhn and colleagues, and Siegler and colleagues), any transition from the spatial strategy to cancellation is generally rapid and complete.
Roberts et al. (1997) investigated several potential predictors of strategy usage (verbal ability, spatial ability, intelligence, knowledge of compass points) and found that by far the most important was performance on a spatial ability test, which involved mental rotation and folding. However, the relationship was counterintuitive: People with high spatial ability showed a strong tendency to use cancellation, whereas people with low spatial ability tended to use the spatial strategy (see also Cooper \& Mumaw, 1985). If the action of a visualizer-verbalizer cognitive style had determined strategy selection, the reverse would have been expected, although this does not rule out the action of another unspecified style - there are plenty to choose from. Roberts et al. instead interpreted this as showing that while the spatial strategy is available to all people, cancellation is mainly available to people with high spatial ability. The suggested reason for this was that people with high spatial ability would be better able to construct an accurate, stable representation of each path, from which regularities could be detected (opposite directions canccl no matter how many steps intervene) and redundant steps deleted (the entire construction of the spatial representation). For people with low spatial ability, the higher degree of "representational noise" would make regularities far less likely to be present and detectable. In other words, a person who finds that taking steps east, north, and west places him or her due north for some trials (correctly) and due north northeast for others (incorrectly) is far less likely to discover that cancellation is a viable strategy. The low spatials were hence trapped with a strategy that they were particularly ill-equipped to execute.

The general principle of ability-constrained redundancy deletion is potentially of great importance and is likely to apply to many domains. For example, children with poor numerical skills may obtain different answers to the same arithmetic problems repeatedly. They will hence have difficulty either simplifying procedures, or committing solutions to memory (Lemaire \& Siegler, 1995). However, there is a knowledge-based explanation of the findings of Roberts et al. (1997), implied by Lohman and Kyllonen (1983), who found that the best performers at spatial ability test-type items were those who applied the widest range of strategies and were not necessarily utilizing superior spatial representations. Hence, for Roberts et al., high spatial people who used cancellation may simply have possessed a larger repertoire of strategies for spatial tasks, gained from appropriate past experience.

\section{EXPERIMENT 1}

In Experiment 1, we aimed to test a number of issues arising from Roberts et al. (1997) and elsewhere. Subjects were given different pretasks followed by a standard directions task, and their strategy usage was compared with that of a baseline group. Subjects in the instructed group were given two blocks of directions task trials. For the first, they were instructed to use cancellation in order 
to solve them. For the second, they were given a free choice of strategy. The purpose of this condition was to investigate further the concept of cognitive style as a general account of strategy selection. If the use of the spatial strategy represents a stylistic preference, we should expect people to revert back to this in Block 2. If this use instead reflects the lack of availability of cancellation then, having made this available in Block 1 , we would expect the majority to remain with cancellation when given a free choice.

Subjects in the dax/med group were given one block of $\mathrm{dax} / \mathrm{med}$ word puzzles followed by one block of directions task trials. Each dax/med trial consisted of strings of the words dax (equivalent to east); med (west); slok (north); and rits (south). In any string, dax cancelled med, and slok cancelled rits, so that [slok dax rits rits dax] reduced to [dax rits dax] - equivalent to east southeast. The task is isomorphic with the directions task, although spatial descriptions, and the words cancellation and opposite, were not used when we explained it. Pilot studies had shown that subjects were able to understand the task, solve the problems by using cancellation, and perform no worse than when given a directions task. The purpose of this condition was to investigate further the knowledgebased account of why cancellation is used only by some for the directions task. It is unlikely that even those who use cancellation have encountered the task before, and so this explanation must assume that appropriate knowledge resulted from past experience with related tasks. This explanation is unfalsifiable because we cannot know people's precise past histories. However, if subjects solving the dax/med task were then more likely to use cancellation for the directions task, this would suggest that such an account is at least plausible.

Subjects in the paper \& pencil group were given two blocks of directions task trials. For the first, they were given the option of using pencil and paper to solve the problems. For the second, these were unexpectedly removed. The purpose of this condition was to test the suggestion by Crowley et al. (1997) that strategy development takes place as a result of a competitive negotiation between metacognitive and associative mechanisms (this has been modeled successfully by Shrager \& Siegler, 1998). Here, an unpracticed task places considerable demand on metacognitive processes, but with practice, low-level mechanisms are able to take over and work unsupervised. The freed metacognitive resources may then be used to search for regularities and redundancies in the current strategy. Any identified improvements can then be implemented by redirecting the low-level processes toward executing them. Hence, new strategies are more likely to be discovered when metacognitive resources are freed. If we assume that the use of pencil and paper to solve directions task problems frees metacognitive resources, we might expect the increased discovery of cancellation. However, given that pencil and paper would make the spatial strategy easier to execute, this might also reduce the incentive to select cancellation. Subsequent removal of the pencil and paper should then encourage cancellation to be selected in preference.

\section{Method}

Subjects. Eighty students from the University of Essex were pretested with the Saville and Holdsworth Advanced Test Battery ST7 Spatial Reasoning test (Saville \& Holdsworth Ltd, 1979). This requires people to fold 2-dimensional figures mentally in order to make patterned cubes, and then decide which of various options they correspond to. Spatial ability was matched for the four groups. There were 20 subjects in each: baseline (mean spatial score $=14.8$, $S D=6.7 ; 9$ males, 11 females); pencil \& paper (mean spatial score $=$ $15.5, S D=6.3 ; 4$ males, 16 females); instructed (mean spatial score $=15.1, S D=7.5 ; 9$ males, 11 females); and dax $/$ med (mean spatial score $=15.3, S D=6.3 ; 6$ males, 14 females). All subjects were naive with respect to the tasks and received either a course credit or a payment of $£ 4$.

Apparatus. Stimuli were presented by an Apple Power Macintosh $7200 / 90$ microcomputer running PsyScope (Cohen, MacWhinney, Flatt, \& Provost, 1993).

Materials. All groups first received 24 trials of a compass point naming task. Each trial consisted of a bold cross on the center of the screen with a diamond in the vicinity. There were two trials for each compass point, excluding the cardinal points. The directions task consisted of one practice and two sets of 18 experimental trials, the latter consisting of six each of six-, seven-, and eight-step problems. For each trial, all steps were shown vertically simultaneously on one screen. No adjacent steps were opposites, and the path ended either on one of the eight simple compass points (e.g., N, S, NE, NW), or near one of the eight complex points (e.g., NNE, ENE). The dax/ med task consisted of one practice and two sets of 18 word puzzle problems. Each set consisted of trials equivalent to those used for the direction task such that the compass point names were replaced as follows: $d a x=$ east; med $=$ west; slok $=$ north; and rits $=$ south. Word strings were presented horizontally.

Design. The experiment was primarily a between-subjects design in which the Block 1 task was the independent variable. This comprised dax/med puzzle trials, standard directions task trials (the baseline group), directions task trials with the option to use pencil and paper, or directions task trials with instructions to use cancellation. The dependent variable was the reported strategy for Block 2 . To demonstrate the validity of the verbal reports, the strategy described for the directions task was also used as a classification variable, with solution time and error rate as the dependent variables.

Procedure. The subjects were tested individually in a single session lasting up to approximately $30 \mathrm{~min}$. Written instructions emphasizing speed and accuracy were given before each task. The instructions for the compass point naming task additionally included a diagram showing the 16 compass points. Each of its trials began with a blank screen shown for $1 \mathrm{sec}$, followed by a light fixation cross shown for another second. The trial was then presented. When the subject responded, the experimenter began the next trial. If no response was made within $10 \mathrm{sec}$, the next trial commenced automatically.

For the baseline, pencil \& paper, and instructed groups, the directions task was administered next. For the instructed group, the cancellation strategy was also described. The subjects were told that it was essential that they should solve every trial by canceling out opposite directions, and that they should resist any temptation to trace the path in any way. They were reassured that this was a valid strategy. For the pencil \& paper group, blank sheets of paper and pencils were provided. The subjects were informed that they were free to use these if they felt that this would improve their performance. All three groups first attempted the practice trial and were then shown a diagram of its path along with its answer. The Block 1 tri- 
als then followed. After this, the subjects were given a short break and the pencil \& paper group were informed that they would no longer have this option. The instructed group were informed that they were no longer required to use cancellation. All groups were then given the Block 2 trials. The two sets of directions task trials were counterbalanced.

For the dax/med task, the subjects were informed that they should reduce each word string and express it in its simplest form by applying certain rules, which were not explicitly described. They were told that slok was linked to rits, and dax was linked to med. They were taught the task by being given numerous examples, with increasing numbers of steps, of the rules in action. For example, "dax slok med becomes slok" and "dax dax dax rits med rits med becomes rits rits dax." The words opposite and cancel were never used during the instructions. The subjects were requested to give answers analogous to those for the directions task. For example, if slok, dax, and slok remained, the required answer was slok slok dax (NNE). These were taught by example before the task, and a listing of the 16 permitted permutations was available for consultation between trials. The practice trial followed and was explained, and then one of the sets of experimental trials was presented. Next, there was a short break during which the subject was given instructions for the directions task, a practice trial, its answer, and the complementary set of problems. Hence, if the dax/med puzzles had been derived from one set of directions task trials, the other set of directions task trials was presented.

Each trial for the directions task and the dax/med puzzles was preceded by a fixation' cross which remained on the screen for $1 \mathrm{sec}$. The trial remained on the screen until the subject responded verbally. On commencement of speaking, the experimenter clicked the mouse, which caused the computer to remove the trial, display a blank screen, and record the solution time. The experimenter noted the response, and commenced the next trial when subjects indicated that they were ready to continue. The order of presentation within a set of trials was randomized. At the end of the tasks, the subjects were asked to describe in writing the strategies, or combination of strategies, that they had used for the directions task (and the dax/med task if appropriate). People in the instructed group were additionally asked to reread the cancellation instructions and then fill in two five-point rating scales estimating (1) the extent to which they had been able to follow these for the majority of trials and (2) the proportion of trials for which they had been able to follow these exactly.

\section{Results}

Absolute error rates are reported throughout. Permutation slips were not counted as errors. For example, answers of "ENN" or "dax slok slok" were taken as NNE or slok slok dax, respectively. In order to have sufficient data points to calculate accurate means, solution times were included for answers that were no more than one compass point out. The analysis of performance and strategy usage considers the Block 2 trials only. The written reports were classified in two ways, by the experimenters, after the test sessions. The dominant strategy was the one reported as being used for the majority of trials. We used this classification when we analyzed performance. To investigate strategy selection, we also classified reports by final choice of strategy. This distinction was necessary, since some subjects selected cancellation only toward the end of the trials, and thus the spatial strategy would dominate performance. The subjects were able to report their chosen strategies with little difficulty. Where two strategies were described, most reported changing with a clean break. For consistency, those who claimed to have used both strategies for an equal number of trials were classified as spatial strategy dominant. Only 3

Table 1

Mean Performance and Dominant and Final Choice of Strategy for Experiment 1

\begin{tabular}{|c|c|c|c|c|}
\hline & \multicolumn{4}{|c|}{ Group } \\
\hline & Baseline & Instructed & Pencil \& Paper & Dax/Med \\
\hline \multicolumn{5}{|c|}{ Performance } \\
\hline Block 1 task & Standard & Instructed & Pencil \& Paper & Dax/Med \\
\hline \multicolumn{5}{|c|}{ Block 1 solution time $(\mathrm{sec})$} \\
\hline$M$ & 12.4 & 8.9 & 12.3 & 10.9 \\
\hline$S D$ & 7.0 & 2.9 & 5.6 & 3.6 \\
\hline \multicolumn{5}{|c|}{ Block 1 percentage error } \\
\hline$M$ & 51.5 & 33.4 & 42.2 & 33.6 \\
\hline$S D$ & 24.2 & 19.0 & 24.2 & 23.0 \\
\hline Block 2 task & Standard & Standard & Standard & Standard \\
\hline \multicolumn{5}{|c|}{ Block 2 solution time ( $\mathrm{sec}$ ) } \\
\hline$M$ & 10.9 & 7.4 & 9.8 & 12.5 \\
\hline$S D$ & 5.0 & 2.2 & 3.1 & 5.0 \\
\hline \multicolumn{5}{|c|}{ Block 2 percentage error } \\
\hline$M$ & 49.5 & 32.0 & 50.9 & 46.8 \\
\hline$S D$ & 28.2 & 18.2 & 22.0 & 19.0 \\
\hline \multicolumn{5}{|c|}{ Block 2 Strategy Usage } \\
\hline \multicolumn{5}{|c|}{ Dominant strategy } \\
\hline Spatial & 14 & 1 & 15 & 14 \\
\hline Cancellation & 6 & 19 & 5 & 6 \\
\hline \multicolumn{5}{|c|}{ Final choice of strategy } \\
\hline Spatial & 12 & 1 & 13 & 13 \\
\hline Cancellation & 8 & 19 & 7 & 7 \\
\hline
\end{tabular}


of the 80 subjects were difficult to assign to a particular strategy; these reported having used a mixture throughout the trials. For example, a subject might report having used the spatial strategy for the majority of trials, but occasionally using the other to double check. Here, the majority strategy was taken to be both dominant and the final choice. Summary statistics are given in Table 1.

For Block 1 of the instructed group, everyone indicated that they had been able to follow the instructions closely for at least the majority of trials. Most indicated that they had been able to follow them exactly for all trials. Only one subject in this group changed to the spatial strategy for Block 2. When comparing performance for the two blocks, we found this to be adaptive. For Block 1, there was a mean solution time of $11.5 \mathrm{sec}(S D=5.1)$ per problem versus a mean solution time of $8.2 \sec (S D=5.6)$ per problem for Block 2, and this difference was significant $(U=45, p<.05)$. Block 2 errors ( 8 from 18 trials) were also fewer than for Block 1 ( 10 errors) but not significantly so $\left[\chi^{2}(1)=0.44, p>.05\right]$.

For Block 2, 44 subjects overall were classified as spatial and 36 as using cancellation as their dominant strategy. Spatial strategy users were slower with a mean solution time per problem of $12.0 \mathrm{sec}(S D=4.6)$ versus $7.8 \sec (S D=2.6)$ for cancellation users $(U=273, p<$ $.01)$. Spatial strategy users were also less accurate (mean error rate $=58.5 \%, S D=17.2$ ) than cancellation users (mean error rate $=28.0 \%, S D=17.6 ; U=181, p<.01$ ). The relationship between strategy, solution time, and error rate can be seen in Figure 1. By confirming the expected patterns of performance, this analysis shows that people were able to report their strategies accurately. The mean solution time and error rate for the dax/med task lie between the levels of performance for cancellation and spatial strategy dominant subjects for the directions task. This suggests that the dax/med task was not the more dif- ficult. Indeed, compared with people who used the spatial strategy for the directions task, the dax/med task appears to have been easier.

An overall $\chi^{2}$ analysis of final choice of strategy revealed a significant difference in strategy usage across groups $\left[\chi^{2}(3)=20.6, p<.01\right]$. Comparing each group against the baseline yielded a significant difference for the instructed group $\left[\chi^{2}(1)=13.8, p<.01\right]$, and no significant difference for either the pencil \& paper or the dax $/$ med groups $\left[\chi^{2}(1)=0.107, p>.05\right.$ for both $]$. Thus, only for the instructed group was there evidence of any increase in cancellation in Block 2 compared with the baseline.

\section{Discussion}

For the instructed group, cancellation was the preferred strategy when a free choice was subsequently permitted. This shows that where people use the spatial strategy under normal circumstances, virtually none do so out of choice, and so the patterns of strategy usage observed here cannot have been stylistic. For the dax/med group, there was no transfer of cancellation to the directions task. This finding by itself cannot rule out a knowledge-based account of strategy usage. Perhaps there was insufficient practice at the dax/med task, although people were often more proficient at this than at the directions task. Alternatively, perhaps the dax/med task was insufficiently similar to the directions task, although one is then forced to wonder just how similar two immediately adjacent tasks must be for strategies to transfer from one to the other. These results show that to assert a knowledge-based account of strategy usage is by no means trivial. Appealing to the past as an explanation of current performance requires corroborating evidence at the very least. Ideally, specification would be needed of exactly which past knowledge might lead to the use of cancellation at the di-

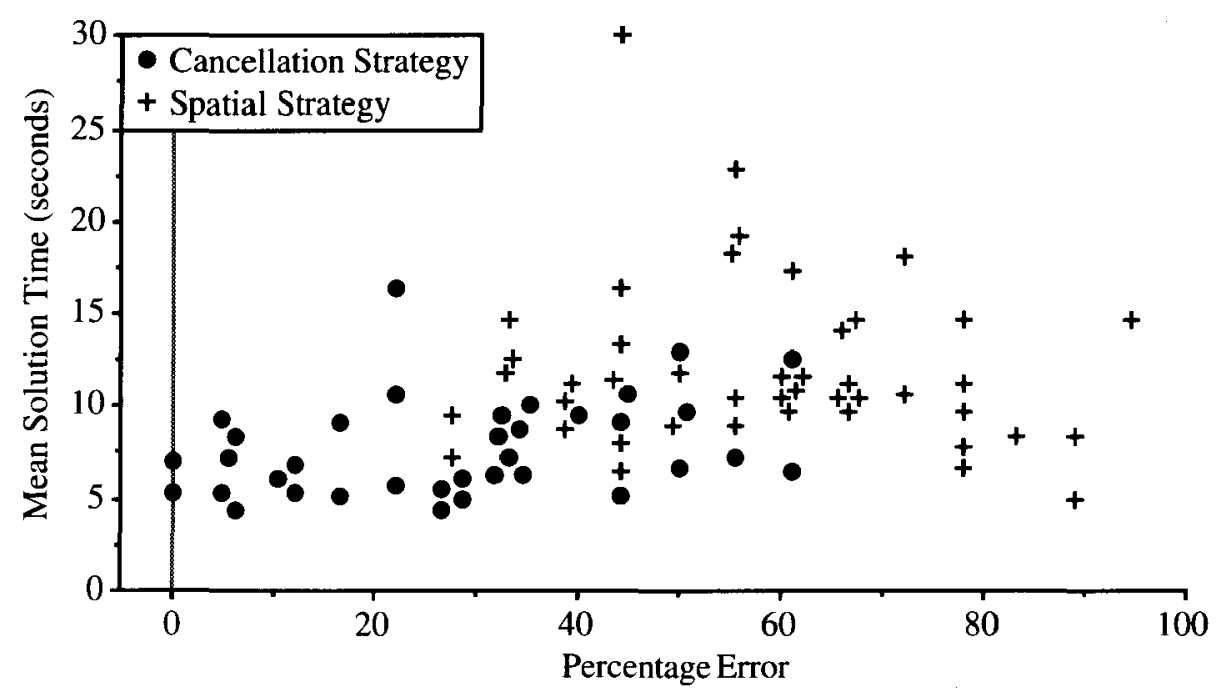

Figure 1. Scattergram of mean solution time versus error rate for the directions task: Experiment 1, Block 2. 
rections task. Finally, for the pencil \& paper group, there was no increase in cancellation. This could be because pencil and paper do not free metacognitive resources sufficiently for new strategies to be discovered. However, if this is the case, it is hard to see why subjects would adopt pencil and paper so enthusiastically, nor why the majority should have expressed irritation when the option was taken away. Instead, perhaps Crowley et al.'s (1997) theory needs qualification: As metacognitive resources are freed, strategy discovery becomes potentially more likely, but this is tempered because the need to search for alternatives becomes simultaneously less necessary.

\section{EXPERIMENT 2}

Experiment 1 had shown no evidence for the transfer of cancellation between tasks, hence casting doubt on the generality of a knowledge-based account of strategy usage. However, it was still possible that users of cancellation for the directions task already knew that this would be a viable strategy from past experience and that, for whatever reason, possession of this knowledge was associated with high spatial ability. A further study by Roberts et al. (1997) is relevant here. They found that the option of using pencil and paper vastly reduced the incidence of cancellation and lowered performance as a result. This could be explained either by pencil and paper suppressing the need to search for and discover alternative strategies, or by pencil and paper suppressing the need to use alternatives. Hence, for the latter, some people may bring cancellation to this task in their strategy repertoires, but the option to use pencil and paper means that they perceive no need to use it. Although Roberts et al. preferred the discovery suppression account, this was an ad hoc explanation for unexpected results. In Experiment 2, we aimed to identify the better of these alternatives, further testing the knowledge-based account. The subjects in the instructed group were given two blocks of directions task trials. For Block 1, they were instructed to use cancellation in order to solve them. For Block 2, they were given a free choice of strategy with the option to use pencil and paper. The baseline group received two blocks of directions task trials. There were no strategy instructions, and the option to use paper and pencil was available throughout. For Block 2, if the instructed group were to remain with cancellation, this would show that pencil and paper do not suppress an effective strategy already present in the repertoire. Instead, this would show that the results of Roberts et al. were due to the suppression of strategy discovery.

\footnotetext{
Method

Subjects. Forty students from the University of Essex were recruited and paid identically to those in Experiment 1 . They were pretested for spatial ability and matched as before. There were 20 subjects in each group: pencil \& paper (mean spatial score $=$ $15.3, S D=7.6 ; 9$ males, 11 females), and instructed (mean spatial score $=15.4, S D=7.7 ; 10$ males, 10 female) .
}

Apparatus and Materials. These were identical to those in Experiment 1 , but the dax/med task was excluded.

Design. The experiment was a between-subjects design. The independent variable was the Block 1 task, either with instructions to use cancellation, or with the option to use pencil and paper (the baseline condition). The dependent variable was the final choice of strategy for Block 2 .

Procedure. This was identical to the general format of Experiment 1 , with the following exceptions. For the Block 1 trials, the instructed group had cancellation described to them in the same way as before, and they were requested to use this for every trial. They were not given the option to use pencil and paper until Block 2, where they were free to use any strategy. The baseline group were given the option to use pencil and paper throughout.

\section{Results and Discussion}

Summary statistics are shown in Table 2. For Block 2, 39 of the subjects had a clear dominant strategy, which matched their final choice. One subject-in the baseline group-reported mixing strategies and was classified as using the spatial strategy by the criteria described for Experiment 1. Two subjects in the instructed group used pencil and paper as an aid to cancellation. There was a significant difference in strategy usage between groups for the Block 2 trials $\left[\chi^{2}(1)=40, p<.01\right]$. The subjects who practiced with cancellation remained with this strategy even when given the option to use pencil and paper. This shows that once effective strategies are available, their use is not suppressed by pencil and paper, so that any suppression must occur for their discovery. Thus far, the knowledge-based account of strategy usage has not fared well, and it is clear that this cannot be applied indiscriminately. If we wish to understand individual dif-

Table 2

Mean Performance and Dominant/Final Choice of Strategy for Experiment 2

\begin{tabular}{|c|c|c|}
\hline & \multicolumn{2}{|c|}{ Group } \\
\hline & Baseline & Instructed \\
\hline \multicolumn{3}{|c|}{ Performance } \\
\hline Block 1 task & Pencil \& Paper & Instructed \\
\hline $\begin{array}{l}\text { Block } 1 \text { solution } \\
\quad M \\
S D\end{array}$ & $\begin{array}{r}14.6 \\
4.5\end{array}$ & $\begin{array}{l}8.9 \\
3.8\end{array}$ \\
\hline $\begin{array}{l}\text { Block } 1 \text { percent } \\
\quad M \\
S D\end{array}$ & $\begin{array}{l}40.4 \\
21.9\end{array}$ & $\begin{array}{l}37.0 \\
22.1\end{array}$ \\
\hline Block 2 task & Pencil \& Paper & Pencil \& Paper \\
\hline $\begin{array}{l}\text { Block } 2 \text { solution } \\
\quad M \\
S D\end{array}$ & $\begin{array}{r}13.5 \\
6.6\end{array}$ & $\begin{array}{l}8.2 \\
4.9\end{array}$ \\
\hline $\begin{array}{l}\text { Block } 2 \text { percent } \\
\quad M \\
S D\end{array}$ & $\begin{array}{l}36.1 \\
19.5\end{array}$ & $\begin{array}{l}32.8 \\
21.6\end{array}$ \\
\hline & tegy Usage & \\
\hline $\begin{array}{l}\text { Dominant/final } \\
\text { Spatial } \\
\text { Cancellation }\end{array}$ & $\begin{array}{r}20 \\
0\end{array}$ & $\begin{array}{r}0 \\
20\end{array}$ \\
\hline
\end{tabular}


ferences in strategy usage, it is necessary to go beyond knowledge.

\section{EXPERIMENT 3}

Roberts et al. (1997) suggested two possible means by which low spatial ability prevents the use of cancellation. First, people with low spatial ability are less able to discover it. The second was based on the claim by some subjects that they had considered cancellation but rejected it as invalid. Hence, for some people, there may be a need to evaluate the validity of newly discovered strategies before selection. One way in which a new strategy of unknown validity can be evaluated is to compare its output with that of a strategy that is known to be valid. If the two outputs agree, then the new strategy is also valid. Again, this procedure is easiest for those who can execute the original strategy accurately; otherwise there will be persistent disagreement, and by the logic of this evaluation, the new strategy must be rejected. At first sight, this account is in conflict with Siegler's (e.g., 1996) suggestion that if current strategies are valid, new strategies derived from them will also be valid (this suggestion is conceptualized as the goal sketch). For example, children competent at addition were never observed to use pathological strategies (Siegler \& Jenkins, 1989). Young children were also able to evaluate the elegance of strategies too demanding for them to use (e.g., Siegler \& Crowley, 1994) as well as strategies discovered for themselves (e.g., Siegler \& Jenkins, 1989). If children are capable of this, why is this not the case with university students for the equally simple directions task? One pointer is from Siegler and Jenkins, who found that newly discovered addition strategies were more likely to be generalized rapidly if there was a high degree of understanding of their elegance, perhaps also suggesting the need for evaluation as part of the strategy development process, and individual differences in people's ability to achieve this.

The aim of Experiment 3 was to demonstrate that the need to evaluate a new strategy can be an important obstacle to its availability. There were three groups of subjects, all of whom were given directions task trials but with different levels of feedback. For the first group, no feedback was given. For the second, partial feedback was given: whether each answer was correct. For the final group, full feedback was given: For each trial, subjects were either told that they were correct or told the answer. All trials remained on the screen until the subject indicated that he or she was ready for the next. If evaluation along the lines described above is an important component of strategy development, full feedback would be expected to assist a subject in evaluating strategy effectiveness and validity more effectively than partial feedback, hence leading to more development of cancellation during the task. This pattern of results would also go against a knowledge-based account of strategy usage. If feedback implies that current strategies are inadequate, and a new one must be chosen from the repertoire, any type of feed- back should be equally effective in triggering the selection of cancellation. Hence, for such an account to apply, there should be no differences in the selection of cancellation between the two types of feedback.

\section{Method}

Subjects. Seventy-two students from the University of Essex were recruited and paid identically to those in Experiment 1 . They were pretested for spatial ability and matched as before. There were 24 subjects in each group: no feedback (mean spatial score $=15.4$, $S D=6.4 ; 4$ males, 20 females); partial feedback (mean spatial score $=15.1, S D=6.5 ; 9$ males, 15 females $)$; and full feedback (mean spatial score $=15.5, S D=6.5 ; 6$ males, 18 females).

Apparatus and Materials. These were identical to those in Experiment 1 , but the dax/med task was excluded.

Design. The experiment was primarily a between-subjects design. The independent variable was the level of feedback (none, partial, or full). The main dependent variable was the strategy described by the subjects on the directions task, including whether any change was reported.

Procedure. This was almost identical to that for the baseline group in Experiment 1. The one exception was that after each trial had been answered, it remained on the screen during feedback and until the subjects were ready to continue. This gave an initial solution time and a thinking time for each trial. Depending on the group, the subjects were given no feedback, partial feedback (informed whether correct), or full feedback (informed whether correct, or given the correct response). For the full feedback condition, the experimenter attempted to ensure that the time for feedback for incorrect trials did not exceed that for the partial feedback condition. For each set of trials, one of four prerandomized trial orders was used.

\section{Results}

The same classification method was used as in Experiment 1 . Four subjects mixed strategies throughout; all were classified as spatial strategy users, since no single trial was reported as being solved by cancellation alone. Summary statistics are shown in Table 3. To ex-

Table 3

Mean Performance, Correlations Between Thinking Time and Errors, and Strategy Usage for Experiment 3

\begin{tabular}{|c|c|c|c|}
\hline & \multicolumn{3}{|c|}{ Feedback Group } \\
\hline & None & Partial & Full \\
\hline \multicolumn{4}{|c|}{ Performance (Blocks 1 and 2 Combined) } \\
\hline \multicolumn{4}{|l|}{ Solution time (sec) } \\
\hline$M$ & 12.1 & 14.4 & 13.7 \\
\hline$S D$ & 7.0 & 7.4 & 5.9 \\
\hline \multicolumn{4}{|l|}{ Thinking time (sec) } \\
\hline$M$ & 1.4 & 2.5 & 3.4 \\
\hline$S D$ & 0.7 & 1.1 & 1.6 \\
\hline \multicolumn{4}{|l|}{ Percentage error } \\
\hline$M$ & 53.8 & 45.7 & 38.2 \\
\hline$S D$ & 19.1 & 19.1 & 24.1 \\
\hline \multicolumn{4}{|l|}{ Correlation } \\
\hline$M$ & .10 & .46 & .66 \\
\hline$S D$ & .22 & .18 & .20 \\
\hline \multicolumn{4}{|c|}{ Strategy Usage (Blocks 1 and 2) } \\
\hline Spatial strategy throughout & 21 & 17 & 11 \\
\hline Spatial to cancellation & 0 & 3 & 10 \\
\hline Cancellation throughout & 3 & 4 & 3 \\
\hline
\end{tabular}


amine whether the level of feedback affected the development of cancellation, data were excluded for those who cancelled exclusively from the first trial. Overall, there was a significant difference in the numbers who developed cancellation between groups $\left[\chi^{2}(2)=15.0, p<.01\right]$. There was a significant difference between the full and no feedback groups $\left[\chi^{2}(2)=13.1, p<.01\right]$ and between the partial and full feedback groups $\left[\chi^{2}(1)=5.0, p<.05\right]$. The difference between the partial and no feedback groups was not significant $\left[\chi^{2}(1)=3.4, p=.07\right]$. Thus the level of feedback affected the development of cancellation.

Kruskal-Wallis analysis of variance showed a significant effect of thinking time between groups $[H(2)=30.5$, $p<.01]$. Pairwise comparisons showed that there was a significant difference between the partial and no feedback groups $(U=106.5, p<.01)$ and between the partial and full feedback groups $(U=178, p<.05)$. Next, it was investigated whether the increased group thinking times were evenly distributed across all trials or were linked particularly to errors. This was achieved by looking at the point-biserial correlation, for each subject, between whether or not a trial was an error, and the thinking time for that trial. One subject's data in the full feedback group were excluded since no errors were made. The means of these correlations by group are shown in Table 3 . Kruskal-Wallis analysis of variance showed that there were significant differences between groups $[H(2)=40.8$, $p<.01]$. Pairwise comparisons showed a significant difference between the partial and no feedback groups $(U=$ $58, p<.01)$ and between the partial and full feedback groups $(U=127.5, p<.01)$. Thus the greater the level of feedback, the more that an individual's thinking time was raised when an error was made.

\section{Discussion}

Overall, the results of Experiment 3 show that the development of more effective strategies is most likely in tasks where there is not just an indication that errors have been made (which was found to be of very little benefit) but also information about the answers required instead. Again, these findings go against a knowledge-based account of strategy usage. Hence, rather than feedback triggering the use of an already possessed strategy, cancellation was discovered during the task, and only full feedback enabled an effective evaluation to take place. The results concerning thinking times are also of interest. The mean for the full feedback group was the greatest of all, and subjects were investing this additional time particularly where their answers were in error. Whether this is a necessary part of developing cancellation is open to speculation. At the very least, this indicates that although partial feedback may lead to some metacognitive intervention (cf. Crowley et al., 1997), full feedback increases this considerably for incorrect trials.

For spatial strategy users, what may be taking place during the extended thinking times? Error feedback may indicate the need for an alternative strategy, although there is often an acute awareness of this in any case. If cancel- lation has not been discovered, error feedback may lead to repeating the trial. However, for the people in this position, their spatial representations are such that regularities and redundancies have not been identified thus far. As such, it is unlikely that full feedback can increase the inherent quality of representations, or allow accurate recalibration, in order to identify them. For spatial strategy users who discover cancellation but are uncertain of its validity, incorrect answers may encourage a comparison of the two strategies, leading to the selection of cancellation in preference. Hence, the discovery of cancellation may be insufficient for some people, and evaluation may also be a necessary part of the strategy development process. Overall, a newly discovered strategy may not be selected if its validity is in question. Although strategy discovery may be constrained in such a way that new strategies derived from valid strategies are in themselves valid, people are not necessarily aware that this is the case.

\section{GENERAL DISCUSSION}

The results from Experiment 1 demonstrate that the spatial strategy is rarely selected by choice, and that this occurs when cancellation is not available. Together with the results of Roberts et al. (1997), and taking the arguments of Roberts and Newton (in press), these suggest that stylistic accounts are inadequate. They can only explain strategy usage post hoc, and predict usage on a taskspecific basis. Even where data particularly imply the action of cognitive styles-for instance, where people persist with disadvantageous strategies-Roberts and Newton suggest that this occurs not because of a preference to sabotage performance deliberately, but because failure to succeed at the current strategy leads to an inability to discover alternatives.

Findings in all three experiments here demonstrate that knowledge-based accounts of strategy usage, which posit that users of more effective strategies must have obtained them as a result of past experience, cannot be enough by themselves to explain all adult behavior. It is easy to overgeneralize findings from the expertise literature and to assert that all individual differences in strategy usage can be accounted for by knowledge and experience; but this goes beyond the evidence. Individual differences in people's ability to represent and manipulate information is also important. In particular, if a strategy can be executed consistently and accurately, it will be far easier to discover more effective methods.

If we look more closely at the role of success in strategy discovery, the importance of the freeing of metacognitive resources (Crowley et al., 1997) is still not clear. Although their freeing may be required, too much freeing may remove the need to discover more effective strategies. It is difficult to operationalize these concepts precisely, but the findings reported here suggest that these issues are worthy of further investigation. In addition, although failure by itself hinders strategy discovery (despite highlighting the need for alternatives), the re- 
sults of Experiment 3 show that an awareness of failure in appropriate circumstances may be an important component of the evaluation and subsequent selection of a newly discovered strategy. Hence, validity evaluation has been shown to be a potentially important stage in the strategy development process.

One important difference between this work and that of Siegler and colleagues is the finding that for arithmetic tasks, newly discovered strategies propagated slowly. For the directions task, cancellation once discovered-and, if necessary, evaluated-is mainly selected rapidly, but this is almost certainly because of the relatively great advantage that it has over its competitor. Were the advantage to be less, no doubt various strategies would ebb and flow in much the same way as for arithmetic. Clearly, one important goal of research is to establish the circumstances in which strategies are generalized more or less rapidly.

Kuhn and colleagues have focused on the strategies used by children in hypothesis-testing tasks and are agnostic as to the importance of success and failure for strategy discovery (e.g., Kuhn \& Pearsall, 1998, p. 243). One distinctive aspect of their research is that strategy expansion has been studied: How are strategies increased in sophistication to cope with task demands? This should be contrasted with the directions task here, and with Siegler's research, which have both focused on strategy reduction: How are strategies simplified to reduce processing demands? Although there are likely to be differences between these, again the principle of success is likely to apply: Current strategies can only be expanded when utilized successfully. This highlights the point that a diverse range of tasks has been studied for the purposes of understanding strategy usage. It is certainly not the intention of the present authors to suggest that the directions task is more representative than others. Although allencompassing theories of individual differences in strategy discovery, evaluation, and selection are the goal, this will only be possible when performance at a wide range of tasks is fully understood.

\section{REFERENCES}

Cohen, J., MacWhinney, B., Flatt, M., \& Provost, J. (1993). PsyScope: An interactive graphic system for designing and controlling experiments in the psychology laboratory using Macintosh computers. Behavior Research Methods, Instruments, \& Computers, 25, 257-271.

COOPer, L. A., \& Mumaw, R. J. (1985). Spatial aptitude. In R. F. Dil- lon (Ed.), Individual differences in cognition (Vol. 2, pp. 67-94). Orlando, FL: Academic Press.

Crowley, K., Shrager, J., \& Siegler, R. S. (1997). Strategy discovery as a competitive negotiation between metacognitive and associative mechanisms. Developmental Review, 17, 462-489.

Ericsson, K. A., \& LehmanN, A. C. (1996). Expert and exceptional performance: Evidence of maximal adaptation to task constraints. Annual Review of Psychology, 47, 273-305.

GalotT, K. M., Baron, J., \& Sabini, J. P. (1986). Individual differences in syllogistic reasoning: Deduction rules or mental models? Journal of Experimental Psychology: General, 115, 16-25.

KARMILoff-Smith, A. (1992). Beyond modularity: A developmental perspective on cognitive science. Cambridge, MA: MIT Press.

Kuhn, D., \& Pearsall, S. (1998). Relations between metastrategic knowledge and strategic performance. Cognitive Development, 13, 227-247.

Lemaire, P., \& Siegler, R. S. (1995). Four aspects of strategic change: Contributions to children's learning of multiplication. Journal of Experimental Psychology: General, 124, 83-97.

Lohman, D. F., \& KyLLONEN, P. C. (1983). Individual differences in solution strategy on spatial tasks. In R. F. Dillon \& R. R. Schmeck (Eds.), Individual differences in cognition (Vol. 1, pp. 105-135). New York: Academic Press.

RIDING, R. J., \& RAYNER, S. (1998), Cognitive styles and learning strategies. London: David Fulton.

RoBERTS, M. J., GilmORE, D. J., \& WoOD, D. J. (1997). Individual differences and strategy selection in reasoning. British Journal of Psychology, 88, 473-492.

ROBERTS, M. J., \& NeWTON, E. J. (in press). Understanding strategy selection. International Journal of Human-Computer Studies.

SAVILle \& HoldSwORTH LTD. (1979). Advanced Test Battery; Manual and user's guide. Thames Ditton, U.K.: Author.

Schiano, D. J., Cooper, L. A., Glaser, R., \& Zhang, H. C. (1989). Highs are to lows as experts are to novices: Individual differences in the representation and solution of standardized figural analogies. Human Performance, 2, 225-248.

Shrager, J., \& Siegler, R. S. (1998). SCADS: A model of children's strategy choices and strategy discoveries. Psychological Science, 9 , 405-410.

SIEGLER, R. S. (1996). Emerging minds: The process of change in children's thinking. New York: Oxford University Press.

SiEgler, R. S., \& CrowlEY, K. (1994). Constraints of learning on nonprivileged domains. Cognitive Psychology, 27, 194-226.

SiEGLER, R. S., \& JENKINS, E. A. (1989). How children discover new strategies. Hillsdale, NJ: Erlbaum.

Simon, H. A. (1990). Invariants of human behavior. Annual Review of Psychology, 41, 1-21.

STERNBERG, R. J. (1997). Are cognitive styles still in style? American Psychologist, 52, 700-712.

VANLEHN, K. (1988). Towards a theory of impasse driven learning. In H. Mandl \& A. Lesgold (Eds.), Learning issues for intelligent tutoring systems (pp. 19-41). New York: Springer-Verlag.

Woon, D. J. (1978). Problem solving - the nature and development of strategies. In G. Underwood (Ed.), Strategies in information processing (pp. 329-356). London: Academic Press.

(Manuscript received October 1, 1998; revision accepted for publication July 4,1999 .) 